\title{
A non-invasive blood viscosity detecting method based on pulse wave
}

\author{
Fei Cui ${ }^{1, *}$, Xiaohui Chen ${ }^{1}$, and Jie Peng ${ }^{1}$ \\ ${ }^{1}$ Nanjing University of Posts and Telecommunications Nanjing, China
}

\begin{abstract}
Keywords: Non-invasive blood viscosity, Pulse wave characteristic parameter, Linear regression.
\end{abstract}

\begin{abstract}
A non-invasive blood viscosity detection method based on pulse wave is proposed. The method establishes a relationship equation between blood viscosity and peripheral resistance, arterial compliance, and pulse wave waveform characteristics by extracting characteristic parameters of human pulse wave. According to the blood viscosity model established by linear regression analysis, the human blood viscosity value is estimated to achieve non-invasive blood viscosity detection. The experimental results show that the blood using this method. The viscosity is in good agreement with the blood viscosity values measured by the blood viscometer.
\end{abstract}

\section{Introduction}

Blood viscosity refers to the resistance formed by the friction between two parallel fluid layers when the blood flows, and the blood viscosity is the most important and basic physiological parameter of blood rheological properties. It has the disease diagnosis, treatment effect observation and prediction. important meaning. Blood viscosity testing is divided into invasive and non-invasive, invasive blood viscosity testing is mainly divided into two categories, one is capillary viscosity measurement, and the other is rotary viscosity measurement. Invasive detection of blood viscosity is the most accurate, and is internationally considered as the gold standard for blood viscosity testing, but it has high technical requirements, complicated operation and easy cross infection.

Non-invasive blood viscosity is mainly detected by biomechanical modeling or pulse wave feature extraction. Biomechanical modeling is the use of the relevant principles of fluid mechanics to approximate human blood vessels into tubes through which fluid flows. The algorithm is too complex and has many parameters, which is susceptible to the differences of individual detection, and the accuracy of detection results is limited. Pulse wave feature extraction is to statistically analyze the blood flow waveform parameters and clinical data, correct the blood viscosity model, and bring the blood flow waveform parameters into the modified model to calculate the blood viscosity value. Clinically verified, both have good statistical significance and high accuracy ${ }^{[1]}$.

\footnotetext{
*Corresponding author: 1021829022@qqq.com
} 
The amplitude and shape of the pulse wave contain many important physiological information of the heart and cardiovascular system. It is important to detect and analyze it, and it is of great significance for the prevention and clinical diagnosis and treatment of cardiovascular disease ${ }^{[2]}$. Therefore, this paper first extracts the characteristic parameters of human pulse wave, establishes the relationship between the extracted characteristic parameters and the peripheral resistance, arterial compliance and pulse wave waveform characteristics, and establishes the blood viscosity model by linear regression analysis. According to the blood viscosity model, the blood viscosity value of the human body can be detected, and the non-invasive blood viscosity detection based on the pulse wave is realized. At the end of the paper, this method is verified and discussed.

\section{Detection method}

\subsection{Principle}

The pulse wave is generated by the heart beat, accompanied by blood flow in the peripheral blood vessels. The human blood circulation system is a large and complex system. The shape of the pulse wave is not only affected by the function of the heart itself, but also when the blood flows through various branches. It is also affected by factors such as the peripheral resistance of the blood vessels and the elasticity of the blood vessel wall ${ }^{[3]}$. The pulse wave contains a large number of human cardiovascular physiological and pathological information, and its amplitude and shape can reflect the physiological and pathological information of human blood viscosity ${ }^{[4]}$. This paper proposes a non-invasive blood viscosity detection method based on pulse wave, aiming at achieving high precision non-invasive blood viscosity detection.

\subsection{Pulse wave feature parameter extraction}

The non-invasive blood viscosity detection method proposed in this paper mainly aims to construct a non-invasive blood viscosity detection model by the relationship between blood viscosity and peripheral resistance, arterial compliance, and pulse wave waveform characteristics. Firstly, the characteristic parameters of pulse wave are extracted. There are many characteristic parameters of pulse wave, mainly including amplitude class, time class, area class and other pulse characteristic parameters ${ }^{[5]}$.

First, the characteristic points of the pulse wave must be detected before the feature parameters can be effectively extracted. This paper selects five pulse wave feature points in a cycle, which mainly reflect the physiological state of the cardiovascular system.

The pulse wave feature points are shown in Figure 1:Point $\mathrm{A}$ is the lowest point of the pulse wave waveform, corresponding to the ventricular systole, that is, the beginning of ejection. Point B is the highest point of the pulse wave waveform, corresponding to the maximum ejection capacity, and the higher the amplitude, the faster the ejection speed. Point $\mathrm{C}$ is the first inflection point of the pulse wave drop branch, which corresponds to the closure of the aortic valve, mainly reflecting the aortic resting emptying time, and is susceptible to peripheral resistance. Point D is the local apex of the pulse wave drop branch, corresponding to the refluxing blood encountering the closed aortic valve, and the maximum amount of ejection after reflection mainly reflects the fluidity of the blood vessel wall. Point $\mathrm{E}$ is the lowest point of the pulse wave drop, corresponding to the end of the ejection period ${ }^{[6]}$. 


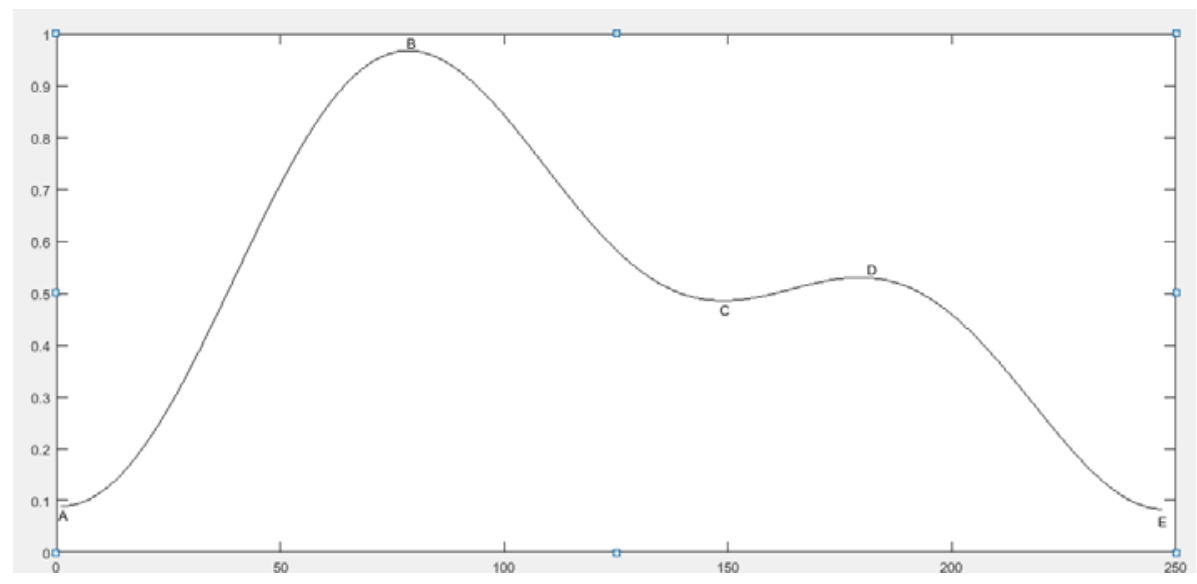

Fig. 1. Feature points of the pulse wave.

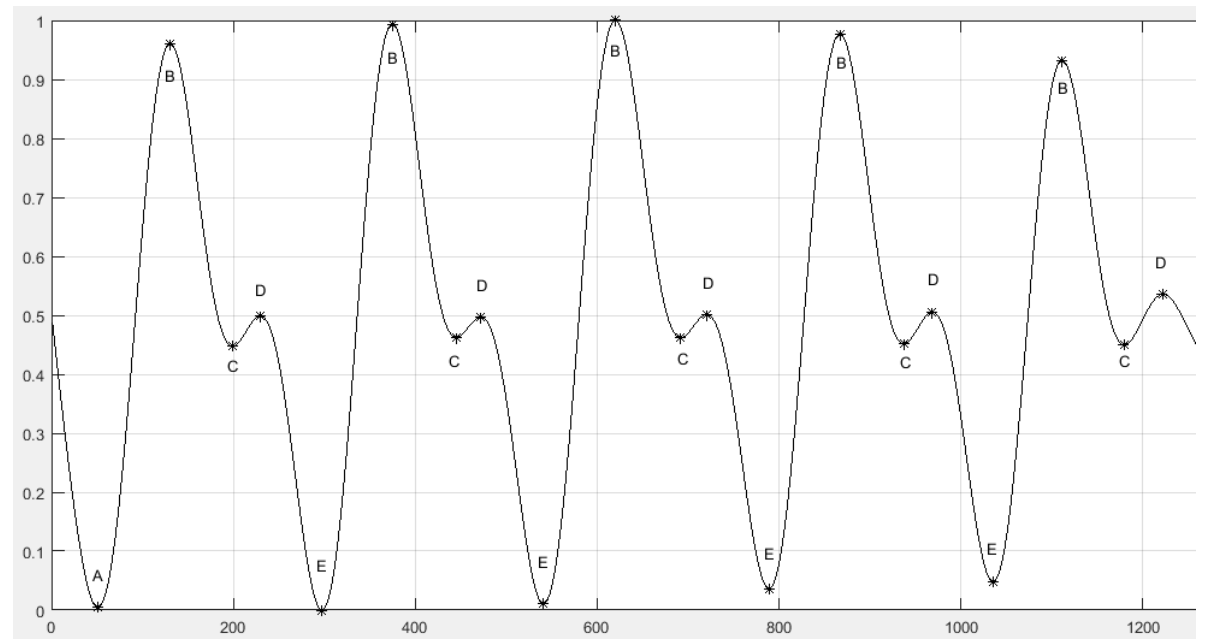

Fig. 2. Pulse wave feature point detection result.

In this paper, the feature points are detected in a step-by-step manner. The feature point detection sequence first describes the feature point detection methods for $\mathrm{A}, \mathrm{B}, \mathrm{E}$, then $\mathrm{C}$ and D.

Firstly, the pulse wave period and the main wave peak point are detected. In this paper, the pulse wave is detected based on the improved extreme value method. Based on the selection of the high quality waveform, the starting point $\mathrm{A}$ and the end point $\mathrm{E}$ of the pulse wave can be determined according to the extreme value method. The pulse wave period, and the maximum point in the detection period is the main wave peak $\mathrm{B}$ point. For point $\mathrm{C}$ and point $\mathrm{D}$, the range interval is determined according to the determined starting point and the main wave peak point, and the extreme point is determined within a specific interval, the maximum point is $\mathrm{D}$ point, and the minimum point is $\mathrm{C}$ point. The detection results are shown in Fig. 2.

After detecting these feature points, the calculation of the pulse wave characteristic parameters is performed. In this paper, five characteristic parameters are selected, namely main wave peak $\mathrm{H} 1$, heavy beat wave valley $\mathrm{H} 2$, heavy beat wave peak $\mathrm{H} 3$, pulse wave period $\mathrm{T}$, and pulse wave waveform characteristic quantity $\mathrm{K}$. Among them, $\mathrm{H} 1$ reflects the aortic compliance function, $\mathrm{H} 2$ reflects the peripheral resistance, and $\mathrm{H} 3$ reflects the blood 
flow $^{[7]} . \mathrm{K}$ is a characteristic of the pulse wave waveform based on the change of the area of the pulse wave, which can reflect the most important parameters of the cardiovascular system such as the peripheral resistance of the blood vessel and the elasticity of the blood vessel wall ${ }^{[8]}$.

\subsection{Establishment of blood viscosity model}

To establish a relationship between blood viscosity and peripheral resistance, arterial compliance, and pulse wave waveform characteristics, we selected 10 health testers aged 20-30 to conduct trials to obtain data. The test procedure is as follows: First, after the tester maintains a stable state, the pulse wave waveform is collected by the photoelectric sensor, and the acquisition time is 1 minute. At the same time, the blood viscosity of the tester is measured by a blood viscometer, and the acquired waveform is preprocessed and selected. A high-quality pulse wave waveform is generated, and feature point detection and feature parameters are extracted therefrom.

According to the above data, using blood viscosity as the dependent variable, the characteristic parameters were converted into peripheral resistance, arterial compliance, and pulse wave waveform characteristic quantities and used as independent variables for linear regression analysis. The results are shown in Table 1.

Tab.1 Blood viscosity linear regression analysis results

\begin{tabular}{|c|c|c|c|}
\hline tester & Pulse wave waveform feature quantity & Peripheral resistance & Arterial compliance \\
\hline 1 & 0.868 & 0.655 & -0.676 \\
\hline$(2)$ & 0.845 & 0.673 & -0.628 \\
\hline$(3)$ & 0.829 & 0.681 & -0.635 \\
\hline$(4)$ & 0.904 & 0.691 & -0.646 \\
\hline (5) & 0.767 & 0.703 & -0.651 \\
\hline$(6)$ & 0.923 & 0.663 & -0.637 \\
\hline$(7)$ & 0.856 & 0.697 & -0.683 \\
\hline$(8)$ & 0.859 & 0.715 & -0.613 \\
\hline$(9)$ & 0.874 & 0.659 & -0.685 \\
\hline$(10$ & 0.862 & 0.689 & -0.701 \\
\hline
\end{tabular}

It can be seen from the table that blood viscosity has a good correlation with peripheral resistance, arterial compliance, and pulse wave waveform characteristics.

\subsection{Blood viscosity estimation method}

According to the correlation between blood viscosity and peripheral resistance, arterial compliance, pulse wave waveform characteristics and the above analysis results, a non-invasive blood viscosity detection method based on pulse wave is proposed.

The first step is to establish a blood viscosity model. Pre-process the acquired waveform, select the high-quality waveform and perform feature point detection and feature parameter extraction. The extracted characteristic parameters were transformed into peripheral resistance, arterial compliance, pulse wave waveform feature quantity, peripheral resistance, arterial compliance, pulse wave waveform characteristic quantity as independent variables, blood viscosity value as dependent variable, and established by linear regression analysis. Equation of relationship between blood viscosity value and peripheral resistance, arterial compliance, and pulse wave waveform characteristic quantities. In the second step, the blood viscosity value is estimated by the blood viscosity model, and the characteristic pulse detection and characteristic parameter extraction are mainly performed on the acquired 
pulse wave waveform, and the characteristic parameters are converted into peripheral resistance, arterial compliance, and pulse wave waveform characteristic quantities are brought into the blood. In the viscosity model, the blood viscosity value is calculated to achieve non-invasive blood viscosity detection.

\section{Experiment analysis}

In order to test the accuracy of the above blood viscosity detection method, we used the Bland-Altman method and the measured blood viscosity value for data analysis. The advantage of the Bland-Altman method is that it can consider both random and systematic errors. It is an intuitive, simple and convenient method for consistency evaluation. Using the above two methods, 30 sets of blood viscosity values were collected separately for data analysis. The results in Figure 3 show that most of the data are in the 95\% confidence interval, so it can be concluded that the two blood viscosity detection methods have good consistency.

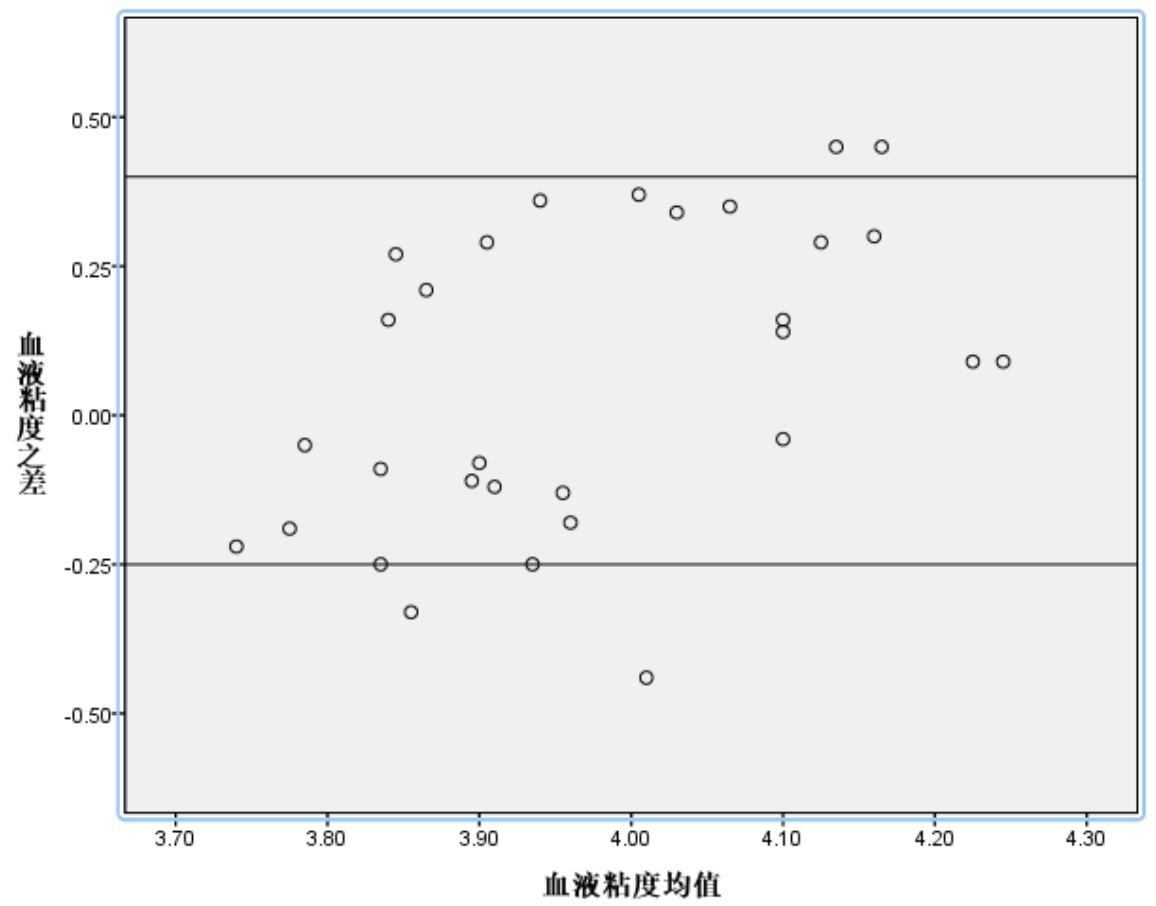

Fig. 3. Bland-Altman diagram of blood viscosity measured by pulse wave blood viscosity test and blood viscometer.

\section{Conclusion}

Based on the analysis of blood viscosity and pulse wave, a non-invasive blood viscosity detection method based on pulse wave is proposed. The detection method mainly extracts the characteristic parameters of the pulse wave of the human body, and converts the characteristic parameters into the peripheral resistance, the arterial compliance, the characteristic waveform of the pulse wave waveform, establishes a blood viscosity model to estimate the blood viscosity value of the human body, thereby realizing the non-invasive blood viscosity detection. According to the analysis results of the Bland-Altman method, 
the blood viscosity value measured by the non-invasive blood viscosity detecting method has good consistency with the blood viscosity value measured by the blood viscometer. The pulse wave-based non-invasive blood viscosity detection method can detect the blood viscosity value of the human body, thereby realizing the detection of non-invasive blood viscosity in a true sense.

\section{References}

1. Dongming Wang. Research on embedded blood viscosity detection system [D]. Beijing University of Technology. 2012.

2. Haizhen Lü, Zhuangzhi Yan, Weijia Lu. A non-invasive continuous blood pressure measurement method based on pulse wave[J]. Chinese Journal of Medical Instrumentation, 2011, 36(2): 169-173.

3. Hongyu Li. Research on non-invasive blood pressure detection technology based on photoelectric volume pulse wave [D]. Xi'an University of Technology. 2017.

4. Lihua Zheng, Jianhong Dou, Xinghua He etal. Improvement and progress of noninvasive blood pressure measurement technology [J]. China Medical Equipment, 2013, 10 (3): 49-52.

5. Xia Tan, Zhong Ji, Yadan Zhang. Study on non-invasive monitoring of continuous blood pressure based on MI-optimized MIV-BP neural network[J].Journal of Vibration and Shock,2019,9(10):71-79.

6. Xuebo Li. Research and implementation of human physiological index detection algorithm based on photoelectric volume pulse wave [D]. Northeastern University. 2011.

7. Yu Cheng. Pulse wave signal detection and data processing methods [D]. Xi'an University of Technology. 2018.

8. Zhichang Luo, Song Zhang, Wenming Yang. Research on Characteristic Information of Pulse Waveform[J]. Journal of Beijing University of Technology, 1996, 22(1): 71-79. 